\section{P053 SERUM AMYLOID A CAN MODULATE NEUTROPHIL SURFACE EXPRESSION OF L-SELECTIN AND INTEGRIN ALPHA M}

${ }^{1} T$ Kuret* ${ }^{*}{ }^{1,2} \mathrm{~K}$ Lakota, ${ }^{1} \mathrm{P}$ Žigon, ${ }^{1} \mathrm{M}$ Ogric, ${ }^{1,2} \mathrm{~S}$ Sodin-Šemrl, ${ }^{1} \mathrm{R}$ Ješe, ${ }^{1,3} \mathrm{~S}$ Cucnik, ${ }^{1,4} \mathrm{M}$ Tomšic, ${ }^{1} \mathrm{~A}$ Hocevar. ${ }^{1}$ Department of Rheumatology, University Medical Centre Ljubljana, Ljubljana; ${ }^{2}$ FAMNIT, University of Primorska, Koper, ${ }^{3}$ Faculty of Pharmacy; ${ }^{4}$ Faculty of Medicine, University of Ljubljana, Ljubljana, Slovenia

\subsection{6/annrheumdis-2018-EWRR2018.72}

Introduction Serum amyloid A (SAA) is one of the major acute phase proteins, elevated in the sera of newly diagnosed patients with giant cell arteritis (GCA). ${ }^{1}$ SAA was previously shown to activate neutrophils and recently, neutrophils have been recognised as active players in GCA pathogenesis, exhibiting changes in surface protein expression of 1-selectin (CD62L) and integrin $\alpha \mathrm{M}$ (CD11b) during therapy tapering. ${ }^{2}$ Objectives To determine the expression of CD62L and CD11b on neutrophils in peripheral blood of newly diagnosed, steroid naïve GCA patients (day 0) vs. healthy blood donors (HBDs). We also aimed to examine the ability of SAA and SAA1 $\alpha$ to activate neutrophils in whole blood of HBDs and GCA patients prior to receiving glucocorticoid therapy and 7 and 30 days after therapy.

Methods Whole blood of 37 GCA patients and 17 HBDs was stained with anti-CD62L and anti-CD11b (eBioscience), lysed and analysed by flow cytometer (Miltenyi). Whole blood of 5 GCA and $6 \mathrm{HBDs}$ was stimulated with $10 \mu \mathrm{M}$ hrSAA and hrSAA $1 \alpha$ for $20 \mathrm{~min}$ at $37^{\circ} \mathrm{C}$, lysed and incubated on melting ice for $15 \mathrm{~min}$. Samples were then stained with anti-CD62L and anti-CD11b. SAA in sera of GCA patients was measured by nephelometry.

Results We observed higher neutrophil counts and surface expression of CD62L in peripheral blood of naïve GCA patients compared to HBDs $(p=0.006)$. Stimulation of whole blood with hrSAA or hrSAA1 $\alpha$ significantly decreased CD62L and increased CD11b expression on neutrophils of HBDs and naïve GCA patients. Whole blood stimulation with hrSAA/ SAA1 $\alpha$ caused significant attenuation of CD62L, while increasing CD11b expression on neutrophils of naïve GCA patients (day 0) as compared to 7 and 30 days after therapy. Levels of SAA in GCA patients decreased after receiving therapy.

Conclusions We show that hrSAA and hrSAA1 $\alpha$ activate neutrophils implying tight adhesion and transendothelial migration. Interestingly however, in patients with GCA, increased SAA did not shed CD62L, as we observed elevation of both. The latter indicates potential initial attachment of neutrophils to activated endothelium, as a possible mechanism in GCA pathogenesis.

\section{REFERENCES}

1. $0^{\prime}$ Neill $L$, et al. Regulation of inflammation and angiogenesis in giant cell arteritis by acute-phase serum amyloid A. Arthritis Rheum 2015;67:2447-56.

2. Nadkarni $\mathrm{S}$, et al. Investigational analysis reveals a potential role for neutrophils in GCA disease progression. Circ Res 2014;114:242-48.

Acknowledgements The authors would like to thank the Slovenian Research Agency (ARRS) for providing funding for the National Research program P3-0314, as well as the Rotary Club Zgornji Brnik, Slovenia for contributing funding for the flow cytometer. We would also like to thank Prof. Mauro Peretti and Dr. Suchita
Nadkarni from WHRI, Queen Mary, University of London for their support in setting up the protocols.

Disclosure of interest None declared

\section{P054 MONOCYTE-RELATED BIOMARKERS OF RHEUMATOID ARTHRITIS DEVELOPMENT IN UNDIFFERENTIATED ARTHRITIS PATIENTS - A PILOT STUDY}

${ }^{1}$ W Kurowska*, 'E Kuca-Warnawin, ${ }^{1}$ A Radzikowska, ${ }^{2}$ M Maslinska, ${ }^{2} \mathrm{~B}$ Kwiatkowska, ${ }^{1}$ W Maslinski. 'Department of Pathophysiology and Immunology; '2Early Arthritis Clinic, National Institute of Geriatrics, Rheumatology and Rehabilitation, Warsaw, Poland

\subsection{6/annrheumdis-2018-EWRR2018.73}

Introduction The enhanced/disturbed activities of monocytes are important for perpetuation and for development of rheumatoid arthritis (RA). Therefore, consistent analysis of monocytes activities and regulation of molecular pathways operating within monocytes, especially at early stages of RA development, may help to predict the progression to the full-blown disease.

Objectives In this study we aimed to investigate the profile of miRNAs expression in circulating monocytes and monocyterelated cytokines in peripheral blood of individuals at undifferentiated arthritis (UA) stage as potential new biomarkers for RA development.

Methods Magnetically sorted monocytes from peripheral blood (PB) of 20 individuals with UA served for total RNA isolation. RNA samples were used for microRNA profiling performed on the miRCURY LNA array. Concentrations of CCL3/MIP1a, M-CSF, CCL2/MCP-1, IL-6, TNFa, IL-15 and eotaxin in sera of UA patients were measured using commercial ELISA assays. Verification of diagnosis after 4 years of follow-up led to the identification of patients who developed full-blown RA (UA $\rightarrow \mathrm{RA}$ patients) and patients who remained in UA phase (UA $\rightarrow \mathrm{UA}$ patients). Comparisons between patients groups were performed using two tailed Mann Whitney U test.

Results Following computational unsupervised analysis we identified 50 miRNAs in $\mathrm{PB}$ monocytes that have the largest variation of expression across all patients samples. From these 50 miRNAs, expression of three miRNAs: miR-642b-5p $(\mathrm{p}=0.0380)$, miR-483-3 p $(\mathrm{p}=0.009), \operatorname{miR}-371 \mathrm{~b}-5 \mathrm{p} \quad(=0.0381)$ were up-regulated, and two miRNAs: miR-25-3 p (=0.0317) and miR-378d $(p=0.0059)$ were down-regulated in monocytes from $\mathrm{UA} \rightarrow \mathrm{RA}$ vs $\mathrm{UA} \rightarrow \mathrm{UA}$ patients. This specific pattern of miRNAs expression in circulating monocytes paralleled elevated IL-15 $(p=0.003)$ and M-CSF $(p=0.03)$ concentrations in sera of UA patients who progressed to RA.

Conclusions Our results indicate that altered activity of monocytes can be detected at early stages of RA development. We found new miRNA candidates (miR-642b-5p, miR-483-3 p, miR-371b-5p, miR-25-3 p and miR-378d) differentially expressed in $\mathrm{PB}$ monocytes, and elevated concentrations of circulating IL-15 and M-CSF involved in monocyte activity and differentiation, as potential biomarkers identifying UA patients who subsequently developed RA.

Acknowledgements This work was sponsored by grant No: UMO-2011/03/B/NZ6/05035 from National Science Centre 